\title{
Research of mathematical models for minimizing power shortage with quadratic losses in power lines and with using network coefficients (sensitivity coefficients)
}

\author{
Dmitry Krupenev ${ }^{1,2, *}$, Denis Boyarkin ${ }^{1,2}$, and Dmitrii Iakubovskii ${ }^{1}$ \\ ${ }^{1}$ Melentiev Energy Systems Institute of Siberian Branch of the Russian Academy of Sciences, 664033 Irkutsk, Russia \\ ${ }^{2}$ Irkutsk National Research Technical University, 664074 Irkutsk, Russia
}

\begin{abstract}
The article presents an analysis of the models for minimizing the power shortage used in adequacy assessment by the Monte Carlo method. We analysed two models: a model with quadratic losses in power lines and a model using network coefficients (first-order sensitivity coefficients), that are showing the dependence of the change in power flows through power lines on the balance of generation / consumption in the reliability zones. As a result of the analysis, a conclusion is made about the applicability of the investigated models for adequacy assessment in the long-term planning of the development of electric power systems.
\end{abstract}

\section{Introduction}

The adequacy assessment is carried out in the long-term planning of the development of electric power systems (EPS). We used the Monte Carlo method (method of statistical tests) [1-3] to assess the adequacy of large EPSs. The methodology for adequacy assessment based on this method has been developed a long time ago; at present, various components of this method are being enhancement to improve the adequacy of the obtained indicators and make more informed decisions based on them. The methodology for adequacy assessment consists of three main computational stages:

- the stage of the creating the random states of the EPS;

- the stage of determining the volume of power shortage of the EPS;

- the stage of calculating the adequacy indicators of EPS.

Power shortages can arise due to failures of power equipment, an unforeseen increase in demand for power and other factors that are observed during the operation of power systems. Usually, in a while of the adequacy assessment, the minimum total power shortage for the EPS is determined. If we draw a parallel between minimizing the power shortage with real operating conditions, then we can conclude that the operation of the EPS is simulated in terms of the operation of the power system controller while minimizing the power shortage, that is in the event of an emergency situation in the EPS, the actions of the dispatcher are reduced to minimizing the power shortage among consumers.

Modern aspects of the development of power systems, that are consisting in the: development of distributed generation and renewable energy sources; high cost of commissioning and operation of reserves of generating capacities and electric networks; high damage to consumers of electricity and power, due to the occurrence of their deficit, produce the requirements for high accuracy and adequacy of the applied models and methods to minimize the power shortage of EPS.

\section{Models of the minimizing the power shortage of EPS}

The development of models for minimizing the power shortage used in adequacy assessment, and methods for their solution, proceeded gradually as the problem was realized and the progress of mathematical and computational technologies. For today, a number of models have been developed to minimize power shortages. All of them different and have the certain features of the functioning of the EPS, which affect the adequacy of determining the power shortage and other adequacy indicators, as well as the complexity of the power system conception. At the initial stage of development of the methodology for EPS adequacy assessment, the model for minimizing the power shortage was presented in the form of a linear programming problem [4-6]. The first statement of the problem was a linear optimization model without considering power losses during transmission [7]. First time the FordFulkerson algorithm was used for solving this problem. However, this model has a number of disadvantages that affect the accuracy of determining the power shortage in the EPS. There is also the problem of ambiguity in the distribution of the power shortage across the reliability zones, which can significantly distort the real indicators of the EPS adequacy. To eliminate the shortcomings there was proposed a number of modifications of the objective function of the model; similar modifications are presented in [7-9]. The considered models are suitable for a rough

Corresponding author: krupenev@isem.irk.ru 
estimate of the power shortage of EPS for the long-term development of power systems (more than 10-15 years), when the uncertainty in the forecast of the initial data is significant.

Subsequently, the model for minimizing the power shortage was added the power losses during its transmission through inter-zone communications [3-9]. Initially, the power losses were set linearly dependent on the transmitted power through interconnections. It provokes the increase in the accuracy of determining the power shortage in power systems, its distribution over the zones of reliability and the adequacy of the determined indicators of the EPS adequacy.

The next step in increasing the adequacy of the model for minimizing the power shortage was the formulation of the problem, using the quadratic dependence of the magnitude of power losses on the power transmitted through interconnections [4]. The task of minimizing the power shortage of the calculated states of the EPS, with a quadratic loss in the power transmission lines of interzone connections during the transmission of power, can be represented in the following view:

$$
\sum_{i=1}^{I}\left(\bar{l}_{i}-l_{i}\right) \rightarrow \min ,
$$

considering the balance constraints:

$$
\begin{gathered}
p_{i}-l_{i}+\sum_{j=1}^{J}\left(1-k_{j i} f_{j i}\right) f_{j i}-\sum_{j=1}^{J} f_{i j}= \\
0, i=1, \ldots, I, j=1, \ldots, J, i \neq j,
\end{gathered}
$$

and inequality constraints on variables:

$$
\begin{gathered}
0 \leq l_{i} \leq \bar{l}_{i}, i=1, \ldots, I, \\
0 \leq p_{i} \leq \bar{p}_{i}, i=1, \ldots, I, \\
0 \leq f_{j i} \leq \bar{f}_{j i}, 0 \leq f_{i j} \leq \bar{f}_{i j}, i=1, \ldots, I, j \\
=1, \ldots, J, i \neq j, \\
f_{i j} f_{j i}=0
\end{gathered}
$$

where $\bar{l}_{i^{-}}$specified (predicted) value of power consumption in the reliability zone $i, \mathrm{MW} ; l_{i}$ - certain (actual) value of power consumption in the reliability zone $i, \mathrm{MW} ; \bar{p}_{i}$ is the total value of the operating power of generating units in the reliability zone $i, \mathrm{MW} ; p_{i}$ is a certain (actual) value of the loading of generating units in the reliability zone $i, \mathrm{MW} ; \bar{f}_{j i}-$ power connection capacity between zones $j$ and $i, \mathrm{MW} ; \bar{f}_{i j}-$ power connection capacity between zones $i$ and $j, \mathrm{MW} ; f_{j i}$ is the actual load of the connection between zones $j$ and $i$, MW; $f_{i j}$ is the actual load of the connection between zones $i$ and $j$, MW; $k_{j i}$ - coefficients of specific power losses during its transmission from the reliability zone $j$ to the reliability zone $i$.

In this formulation of the problem, there are coefficients of specific power losses in the transmission line during its transmission. The expression for their definition is presented in [4]:

$$
k_{j i}=\frac{r_{j i}}{U_{n o m}^{2} \cos \varphi_{j i}^{2}}, j=1, \ldots J, i=1, \ldots I, i \neq j,
$$

where: $r_{j i}$ is the active resistance of the transmission line between reliability zones $j$ and $i, \mathrm{Om} / \mathrm{km} ; U_{\text {nom }}$ - rated voltage of the power transmission line between reliability zones $j$ and $i, \mathrm{kV} ; \cos \varphi_{j i}$ is the power factor of the transmission line between reliability zones $j$ and $i$ (usually taken equal to 0.9).

Another formulation of the problem of minimizing the power shortage when adequacy assessment is the model using a matrix of network coefficients (MNC) or a firstorder sensitivity matrix, reflecting the dependence of changes in power flows through inter-zone connections on changes in the power balance in the EPS reliability zones [10-13]. The use of matrixes of network coefficients is due to the desire to show the real possible flow distribution of EPS, which depends on many factors, including the reactive resistance of the branches.

In this case, it is required, as in the previous formulation, to determine the minimum power shortage of the EPS, but using the following balance constraints:

$$
\begin{gathered}
f_{i j}=\sum_{i=1}^{I} m_{i j, i} \cdot\left(p_{i}-l_{i}\right), \\
\sum_{i=1}^{I} p_{i}=\sum_{i=1}^{I} l_{i} .
\end{gathered}
$$

where: $m_{i j, i}$ is the coefficient of power flow distribution from the $i$-th zone to the balancing zone for the connection between zones $i$ and $j$ of the EPS, determined by calculations of the steady-state.

An important point in minimizing the power shortage is to take the correct constraints on the variables. So, determining the throughput of inter-zone connections is a rather difficult task, which is solved by calculating the stability of power systems [14]. In the practice of power systems management, it is not inter-zone connections that are consider, but controlled sections, where capacity limitations are calculated for controlled sections by the maximum and emergency permissible active power flows. Inter-zone connections and controlled sections can either coincide in the composition of the power lines included in them, or not. In the case of using restrictions on controlled sections in the problem of minimizing the power shortage, they are used instead of restrictions on inter-zone connections in the above models of the problem of minimizing the power shortage. Most of the compatibility problems of inter-zone connections and controlled sections when minimizing the power shortage in the problem (1) - (6) and (1), (3) - (6), (8), (9) can be avoided by adding a number of conditions into the calculation process, but the problem of accounting for bypass remains unresolved for setting (1) - (6).

The development of both presented models for minimizing the power shortage can be their combination and accounting for both network coefficients and quadratic power losses. In this case, the balance constraint (9) will take the following variant:

$$
\sum_{i=1}^{I} p_{i}=\sum_{i=1}^{I} l_{i}+\sum_{j=1}^{J} k_{j i} f_{j i}^{2} .
$$

In $[11,12]$, a technique for the formation of matrices of network coefficients is presented. The linear model of the power system is used to determine coefficients. It can be represented in matrix form:

$$
\tilde{B} \Theta=P,
$$


where: $\tilde{B}$ - matrix of reactive nodal conductivities (conductivities can be taken in calculations); $\Theta$ and $P$ is the phase angles vectors and active powers of the EPS calculation model nodes respectively; symbols with " $\sim$ denote a diagonal matrix, where the values of a similar vector are located on the matrix diagonal.

Important to attend on the few assumptions: the sine of the angle between the voltage vectors at the ends of the transmission lines is equated to that edge $\left(\sin \theta_{i j}=\theta_{i j}\right)$; voltage losses in the transmission line are neglected and the voltage modules in all nodes of the circuit are equal. After a number of transformations of model (11), an expression is obtained for determining the network coefficients.

$$
M=\widetilde{B}_{V} A^{T} \widetilde{B}^{-1}
$$

where: $\widetilde{B}_{V}$ is the diagonal matrix of reactive conductivities of the EPS branches; $A$ - is the incidence matrix of the EPS nodes calculation model.

We can get the matrix of network coefficients in another more reasonable way. First, it is necessary to write down a system of matrix equations describing the steady state of the EPS only for the active values of the EPS state:

$$
\begin{gathered}
A \bar{S}=S, \quad \bar{S} \in \mathbb{C}^{n}, S \in \mathbb{C}^{m-1}, \\
A^{T} \widetilde{U} U=\tilde{Y}_{V}^{-1} \bar{S}, \quad U \in \mathbb{C}^{m}, \tilde{Y}_{V} \in \mathbb{C}^{n \times n}
\end{gathered}
$$

where: $\bar{S}$ is the vector of active power flows along the branches (transmission lines) of the EPS design model; $U$ is the voltage vector at the nodes of the EPS calculation model; $\tilde{Y}_{V}$ is the diagonal conduction matrix of the EPS branches.

Let us differentiate the system of matrix equations (13) - (14) with respect to $S$ :

$$
\begin{gathered}
A d \bar{S}_{S}=E_{m-1} d S, \\
2 A^{T} \widetilde{U} d U_{S}=\tilde{Y}_{V}^{-1} d \overline{S_{S}} .
\end{gathered}
$$

Let us express from (16):

$$
d \overline{S_{S}}=2 \tilde{Y}_{V} A^{T} \widetilde{U} d U_{S}
$$

Will use (17) in (15):

$$
2 A \tilde{Y}_{V} A^{T} \widetilde{U} d U_{S}=E_{m-1} d S .
$$

Next, let's express from (18):

$$
d U_{S}=\frac{1}{2} \widetilde{U}^{-1}\left(A \tilde{Y}_{V} A^{T}\right)^{-1} d S
$$

Then let's use (19) in (17):

$$
d \bar{S}_{S}=\tilde{Y}_{V} A^{T}\left(A \tilde{Y}_{V} A^{T}\right)^{-1} d S .
$$

From expression (20), we can get the matrix of network coefficients:

$$
M=\tilde{Y}_{V} A^{T}\left(A \widetilde{Y_{V}} A^{T}\right)^{-1}=\tilde{Y}_{V} A^{T} \tilde{Y}^{-1},
$$

where: $A \widetilde{Y_{V}} A^{T}=\tilde{Y}$ is the matrix of nodal conductivities As we can see when comparing expressions (12) and (21), they differ only in that expression (12) uses reactive conductivities, which is accepted initially, and in expression (21), conductivities, otherwise these expressions are identical.

The matrix of network coefficients has the dimension $n \times m-1$ (the number of branches (power lines) by the number of nodes minus the balancing one). For the transformation of $\mathrm{MNC}$ to dimension: the number of controlled sections per the number of safety zones, the coefficients characterizing the EPS nodes are weighted by the share of power consumption in the reliability zone and are added, and the coefficients characterizing the elements of controlled sections are simply added $[11,12]$.

\section{Experimental investigation}

To study the presented models for minimizing the power shortage, calculations were carried out on test scheme: a 3-zone EPS model and IEEE RTS-24 [15]. The diagram of the 3-zone EPS model and the main initial data are presented in Fig. 1 ( $\mathrm{P}$ - generating capacity, L - maximum power consumption, $\mathrm{F}$ - connection transfer capability). Table 1 shows the MNC for the considered EPS scheme. Table 2 shows the results of calculations for the studied models of minimizing the power shortage for the 3-zone EPS.

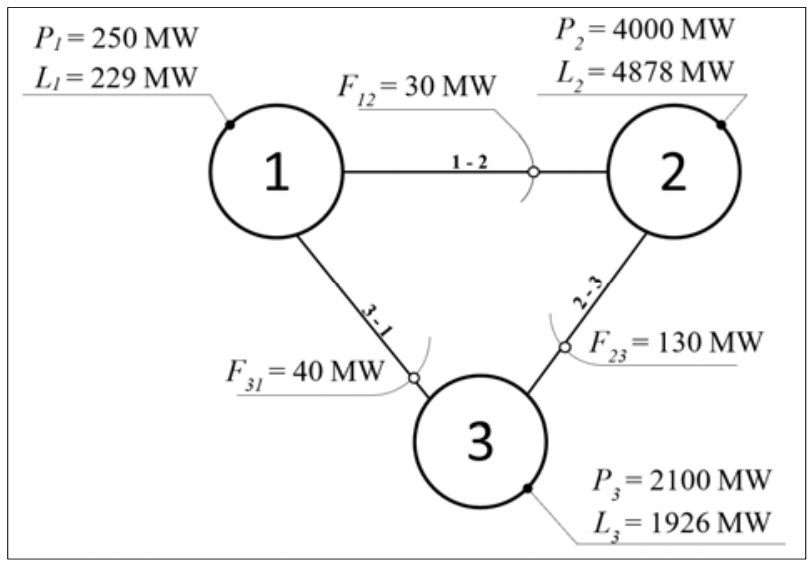

Fig. 1. Scheme and main parameters of the 3-zone EPS model.

Table 1. Matrix of network coefficients of the 3-zone EPS model.

\begin{tabular}{|c|c|c|}
\hline & Reliability zone \#2 & Reliability zone \#3 \\
\hline $\begin{array}{c}\text { Connection } \\
\text { Z1-Z2 }\end{array}$ & $-0,7362$ & $-0,369$ \\
\hline $\begin{array}{c}\text { Connection } \\
\text { Z2-Z3 }\end{array}$ & 0,2638 & $-0,369$ \\
\hline $\begin{array}{c}\text { Connection } \\
\text { Z3-Z1 }\end{array}$ & $-0,2638$ & $-0,631$ \\
\hline
\end{tabular}

It should be noted that the power shortage in the studied EPS was concentrated in the second zone of reliability in all models, this is quite physical and understandable, since the second reliability zone is initially in short supply. As you can see from the table. 2, taking into account the network coefficients influenced 
the total value of the power shortage. So, in the model with quadratic power losses, the value of the power shortage is less than in the other two models, this is the result of additional restrictions imposed by the network coefficients on the transmission of power.

Further studies were carried out on IEEE-RTS-24. The scheme was divided into 5 reliability zones, the division of the scheme and the main initial data are shown in Fig. $2 \mathrm{a}$ and $\mathrm{b}$.

Table 2. Loading of generating units and power shortage in the studied EPS mode.

\begin{tabular}{|c|c|c|c|}
\hline $\begin{array}{l}\text { Reliability } \\
\text { zone }\end{array}$ & $\begin{array}{l}\text { Quadratic } \\
\text { loss model } \\
\text { (1) }\end{array}$ & $\begin{array}{c}\text { MNC } \\
\text { model (2) }\end{array}$ & $\begin{array}{c}\text { MNC } \\
\text { model + } \\
\text { quadratic } \\
\text { loss (3) }\end{array}$ \\
\hline \multicolumn{4}{|c|}{ Generation load, MW } \\
\hline 1 & 250 & 182,9 & 184,6 \\
\hline 2 & 4000 & 4000 & 4000 \\
\hline 3 & 2065 & 2100 & 2100 \\
\hline \multicolumn{4}{|c|}{ Ensured power consumption, MW } \\
\hline 1 & 229 & 229 & 229 \\
\hline 2 & 4159,3 & 4127,9 & 4127,9 \\
\hline 3 & 1926 & 1926 & 1926 \\
\hline \multicolumn{4}{|c|}{ Connection load, MW } \\
\hline 12 & 30 & 30 & 30 \\
\hline 21 & 0 & 0 & 0 \\
\hline 23 & 0 & 0 & 0 \\
\hline 32 & 130 & 97,9 & 97,9 \\
\hline 13 & 0 & 0 & 0 \\
\hline 31 & 9,0 & 76,0 & 76,0 \\
\hline \multicolumn{4}{|c|}{ Reliability zone powers shortage, MW } \\
\hline 1 & 0 & 0 & 0 \\
\hline 2 & 718,7 & 750,0 & 750,0 \\
\hline 3 & 0 & 0 & 0 \\
\hline $\begin{array}{c}\text { EPS power } \\
\text { shortage, } \mathrm{MW}\end{array}$ & 718,7 & 750,0 & 750,0 \\
\hline
\end{tabular}

Table 3. The values of the power shortage in the study of the model with quadratic losses when the mode is heavier, MW.

\begin{tabular}{|c|c|c|c|c|c|}
\hline & Original & $10 \%$ & $20 \%$ & $30 \%$ & $40 \%$ \\
\hline EPS & 0 & 51,76 & 406,47 & 761,71 & 1138,0 \\
\hline Z1 & 0 & 0 & 0 & 0 & 0 \\
\hline Z2 & 0 & 0 & 0 & 0 & 0 \\
\hline Z3 & 0 & 0 & 0 & 0 & 0 \\
\hline Z4 & 0 & 51,757 & 406,47 & 761,71 & 1138 \\
\hline Z5 & 0 & 0 & 0 & 0 & 0 \\
\hline
\end{tabular}

Figure 2 shows the controlled section (S) and their maximum allowable flow. It is worth noting that there are shunts between reliability zones $1-3$ and $4-5$, which are not included in the controlled sections and affect the flow distribution in the EPS.
Tables 3, 4 and 5 show the calculation data for the models under study. In this experiment, the state was weighted with a step of $10 \%$ to increase the maximum power consumption in the reliability zones.

Table 4. The values of the power shortage in the study of the model with the MNC with a heavier mode, MW.

\begin{tabular}{|c|c|c|c|c|c|}
\hline & Original & $10 \%$ & $20 \%$ & $30 \%$ & $40 \%$ \\
\hline EPS & 0 & 216,65 & 526,65 & 837,1 & 1168,23 \\
\hline Z1 & 0 & 0 & 0 & 0 & 0 \\
\hline Z2 & 0 & 0 & 0 & 0 & 0 \\
\hline Z3 & 0 & 137,93 & 327,93 & 518,73 & 746,19 \\
\hline Z4 & 0 & 78,72 & 198,72 & 318,37 & 422,04 \\
\hline Z5 & 0 & 0 & 0 & 0 & 0 \\
\hline
\end{tabular}

Table 5. The values of the power shortage in the study of the model with $\mathrm{MNC}+$ quadratic losses when the mode becomes heavier, MW.

\begin{tabular}{|c|c|c|c|c|c|}
\hline & Original & $10 \%$ & $20 \%$ & $30 \%$ & $40 \%$ \\
\hline EPS & 0 & 216,65 & 526,65 & 837,62 & 1168,74 \\
\hline Z1 & 0 & 0 & 0 & 0 & 0 \\
\hline Z2 & 0 & 0 & 0 & 0 & 0 \\
\hline Z3 & 0 & 137,93 & 327,93 & 519,66 & 747,09 \\
\hline Z4 & 0 & 78,72 & 198,72 & 317,96 & 421,64 \\
\hline Z5 & 0 & 0 & 0 & 0 & 0 \\
\hline
\end{tabular}

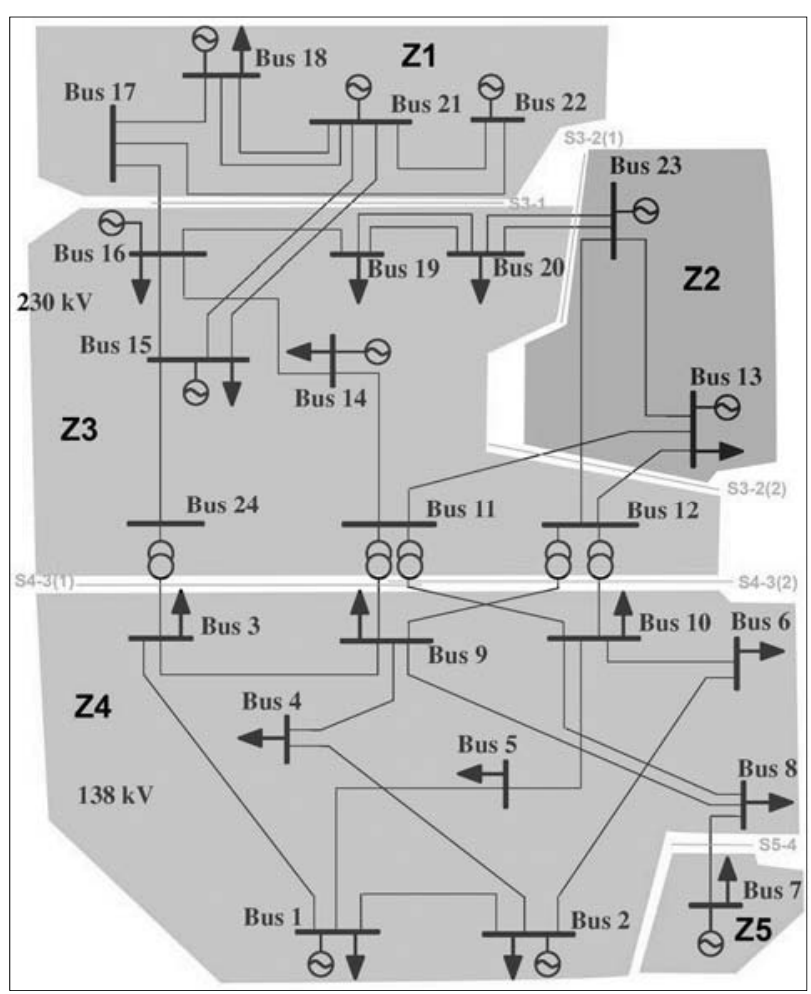

(a) 


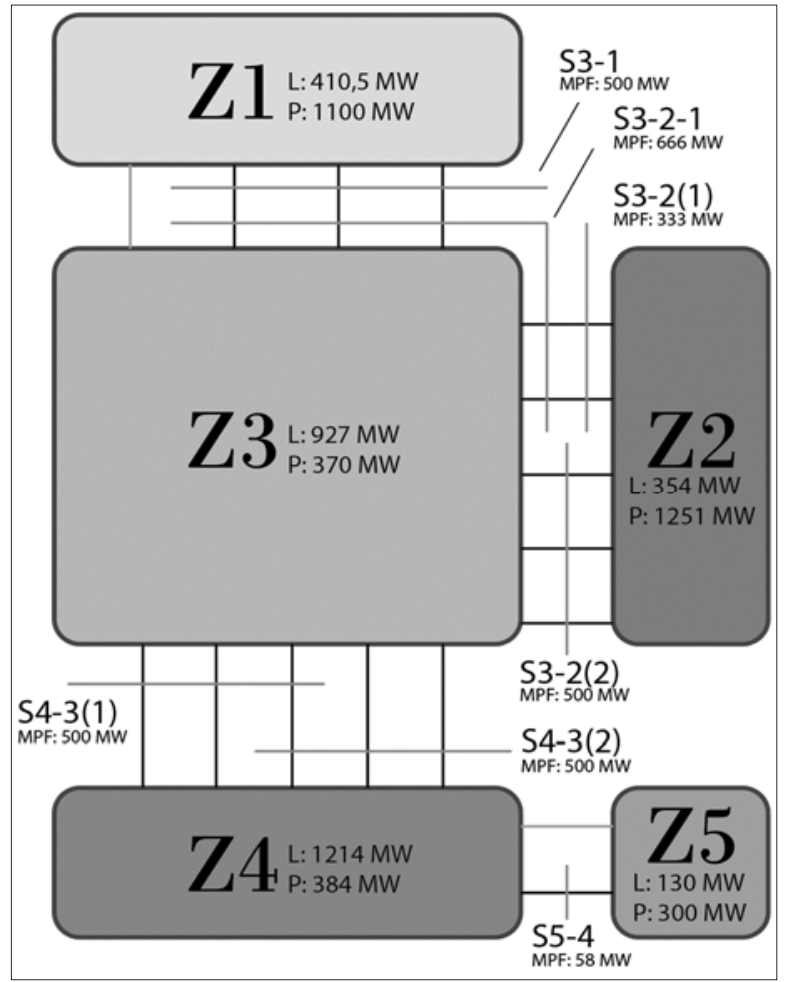

(b)

Fig. 2. IEEE RTS-24 EPS scheme for experimental investigation.

If we analyse the initial situation with the power balance in the reliability zones, then we can conclude that the reliability zones Z3 and Z4 are in short supply. When analysing the power shortage of the initial version of the EPS for all the models under study, it was revealed that the parameters of the EPS network are sufficient to transfer power in the required amount from redundant in terms of generation reliability zones to deficient ones. With an increase in power consumption in the reliability zones by $10 \%$, a power shortage was formed in the calculations for all models, but in the model with quadratic losses the power shortage was formed only in the reliability zone $\mathrm{Z} 4$. In the other two models in the reliability zones Z3 and Z4. At the same time, in the model with quadratic losses, the total value of the power shortage is noticeably lower (51.76 MW) than in other analysed models (216.65 MW). This indicates additional restrictions on power transmission, which arise when taking into account the network coefficients. At the third step of weighting (30\%), a significant difference between the power shortages in the model with quadratic losses and the other two remained, in addition, there appears an insignificant difference as a result between models with $\mathrm{MNC}$ and $\mathrm{MNC}+$ quadratic losses (837.1 MW and 837, $62 \mathrm{MW}$, respectively), this is explained by an increase in the values of the transmitted power along the controlled sections and an increase in power losses during its transmission.

\section{Conclusion}

The adequacy of the model for minimizing the power shortage when assessing adequacy is a necessary requirement for obtaining accurate and adequate indicators of adequacy assessment in the reliability zones. In the future, decisions on the size and locations of reserve generating capacities in the EPS depend on the accuracy of determining the indicators of adequacy.

The work investigated three models for minimizing the power shortage: with quadratic losses; with matrices of network coefficients; with quadratic power losses and matrices of network coefficients. According to the results of the calculation, it can be concluded that the most adequate result is given by a model with matrices of network coefficients + quadratic power losses, since this model takes into account the specifics of the network in terms of power transmission restrictions as much as possible.

The reported study was funded by RFBR, project number 20-0800550_a.

\section{References}

1. Rudenko Yu.N., Cheltsov M.B. Reliability and redundancy in power systems. Research methods. (Novosibirsk: Nauka, 1974)

2. Billinton R., Li W. Reliability Assessment of Electric Power Systems Using Monte Carlo Methods (Springer, 1994)

3. Li W. Probabilistic Transmission System Planning (Wiley-IEEE Press, 2011)

4. Kovalev G. F., Lebedeva L. M. Reliability of Power Systems (Springer Cham; 1st ed. 2019)

5. Rudenko Yu.N., Rozanov M.N., Kovalev G.F. and others Power reserves in the electric power systems of the CMEA member countries. Research methods. (Novosibirsk: Nauka, 1988)

6. Chukreev Yu.Ya. Models for ensuring the reliability of electric power systems (Syktyvkar, 1995)

7. Zorkaltsev V.I., Kovalev G.F., Lebedeva L.M. Investigation of models of power shortage of electric power systems. Bulletin of the Russian Academy of Sciences. Energy. №. 5, (2002)

8. Zorkaltsev V.I., Perzhabinsky S.M. Models for assessing the power shortage of electric power systems. Siberian Journal of Industrial Mathematics. Volume 15. № 1 (49), (2012)

9. Oboskalov V.P. Algorithmic aspects of calculating the probabilistic indicators of the power shortage in the problem of UPS adequacy. Izvestiya RAS. Energy., № 2, (2020)

10. Endreni J. Modeling in reliability calculations in electric power systems. (Moscow: Energoatomizdat, 1983)

11. Belyaev N.A., Korovkin N.V., Chudnyi V.S. Calculation of indicators of adequacy taking into account the variable topology of the electrical network. Electricity, No. 4, (2016)

12. Belyaev N.A., Egorov A.E., Korovkin N.V., Chudnyi V.S. Development of models of electric power 
systems for analyzing the reliability of ensuring the adequacy. Power stations. № 11, (2015)

13. Organization standard 59012820.27.010.005-2018 "Guidelines for carrying out calculations of adequacy."

14. Order of the Ministry of Energy of Russia dated 03.08.2018 No. 630 "Guidelines for the sustainability of energy systems."

15. C. Grigg, P. Wong, etc. The IEEE Reliability Test System - 1996. IEEE Transactions on Power Systems, Vol. 14, №. 3, (1999) 